\title{
Usages et usagers des campus numériques : entre projections ministérielles et prémices d'une réalité
}

Virtual campuses usages and users: between governmental projections and first steps of a reality

Abdel Benchenna et Vincent Brulois

\section{OpenEdition}

Journals

Édition électronique

URL : http://journals.openedition.org/edc/597

DOI : $10.4000 /$ edc. 597

ISSN : 2101-0366

Éditeur

Université Lille-3

Édition imprimée

Date de publication : 1 décembre 2007

Pagination : 133-150

ISBN : 978-2-9514961-8-7

ISSN : 1270-6841

Référence électronique

Abdel Benchenna et Vincent Brulois, « Usages et usagers des campus numériques : entre projections ministérielles et prémices d'une réalité », Études de communication [En ligne], Numéro spécial | 2007. mis en ligne le 01 octobre 2009, consulté le 20 avril 2019. URL : http://journals.openedition.org/ edc/597 ; DOI : 10.4000/edc.597

Ce document a été généré automatiquement le 20 avril 2019

(c) Tous droits réservés 


\title{
Usages et usagers des campus numériques : entre projections ministérielles et prémices d'une réalité
}

\author{
Virtual campuses usages and users: between governmental projections and first \\ steps of a reality
}

Abdel Benchenna et Vincent Brulois

1 Pourquoi s'intéresser aux usages des campus numériques alors que ces derniers ne sont qu'en phase d'expérimentation? Si nous considérons, en effet, que les usages sont des « modes d'utilisation se manifestant avec récurrence, sous la forme d'habitudes relativement intégrées dans la quotidienneté, pour être capables de se reproduire et éventuellement de résister en tant que pratiques spécifiques ou de s'imposer aux pratiques existantes" (Lacroix, Moeglin, Tremblay, 1992), il est difficile de parler d'usages développés sur des campus en train de se constituer. D'autant plus que la durée de vie de certains d'entre eux, sous l'appellation et le format projeté, a été très limitée. Pourtant, loin d'être un frein, cette absence d'usages au contraire rend le sujet intéressant. Après tout, moins que des objets naturels qui se forment par décret, les usages ne représentent-ils pas plutôt un construit social qui s'inscrit dans la durée? Notre intérêt pour cette notion s'explique alors par le fait que nous sommes face à un processus d'innovation qui risque, à long terme, d'être à l'origine de nouveaux usages et éventuellement de nouvelles pratiques résultant des interactions entre acteurs.

Dans ces conditions, une mise en contexte est nécessaire. Celle-ci doit intervenir aussi bien au niveau de la commande ministérielle et des objectifs énoncés (indices d'une réflexion sur les usages) qu'à celui des acteurs et de la traduction qu'ils ont faite de cette commande dans leurs réponses aux trois appels à projets $(2000,2001$ et 2002) ainsi que dans les réalisations sur chacun des campus au fil de leur développement. À chaque 
niveau correspond alors une série de questions, autour de laquelle nous structurerons notre propos.

Ainsi, et dans un premier temps, en quels termes y parle-t-on du (ou des) public(s) visé(s) par ces campus? Une préoccupation pour ce que souhaite ce public apparaît-elle? Il s'agit en fait de comprendre dans quelle mesure l'acteur, à l'initiative de la construction d'une offre de campus, établit pour lui-même et pour autrui une représentation des utilisateurs et des utilisations. Il s'agit encore de chercher des éléments afin de voir dans quelle mesure les usages prescrits peuvent être compris comme une traduction d'exigences politiques à la fois nationale et supranationale. Autre série de questions dans un second temps : comment les consortiums étudiés s'emparent-ils de cet appel ? Quelles offres se sont constituées? Comment ces réponses se traduisent-elles non seulement en dispositifs techniques et en contenus médiatisés, mais aussi en services pédagogiques?

4 L'examen de ces questions nous amènera en conclusion à faire le bilan de ce que l'état actuel des campus numériques en France nous permet de comprendre de l'utilisation des contenus disponibles et des services délivrés, ainsi que de leurs usagers, dans le processus d'innovation en cours.

\section{Projections ministérielles}

\section{Légitimer les actions}

Dès le lancement du projet, le Ministère semble accorder une place de choix aux usages. De la sorte, c'est toute une production discursive qui se met en place, élaborant ainsi une représentation des usages. Par le discours en effet, les promoteurs des campus numériques donnent sens à leur opération. Par la force des choses, ils supposent l'existence de besoins et préconisent des usages. Ce discours n'est pas le fruit du hasard: il est un des éléments d'une stratégie pour intégrer l'innovation souhaitée et s'inscrit dans une démarche volontariste qui entremêle plusieurs enjeux: modernisation de l'université, rayonnement international de la France et de la langue française, développement d'outils de communication. Quatre éléments, notamment, participent à la construction de cette stratégie :

- l'antériorité d'une offre technologique s'appuyant sur les services du réseau Internet (courrier électronique, forum et web) et la présence sur le marché de plates-formes d'enseignement à distance de qualité inégale. Ces possibilités techniques et leur développement sont autant de facteurs qui légitiment un tel discours;

- le développement de pratiques d'enseignement à distance faisant appel aux technologies de l'information et de la communication (TIC) dans l'enseignement supérieur au niveau international et plus particulièrement en Amérique du Nord. L'enjeu ici est à la fois linguistique (place de la langue française dans la diffusion des savoirs), scientifique (place de la recherche française), politique (innovation en matière d'enseignement), voire géopolitique (la place de la France et de la francophonie dans le monde);

- des croyances qui expriment aussi bien des peurs (l'université française serait menacée par la concurrence internationale) que des espoirs (les TIC seraient une des clés de la modernisation). Dans ce contexte, « l'opération Campus » n'en prend que plus d'importance. Il s'agit de "déclencher» une mutation ${ }^{1}$ en invitant les universités à se constituer en consortium pour proposer des projets et à envisager de travailler ensemble, dans la durée ; 
- la traduction d'orientations nationales et supranationales trouvant leur source dans les décisions prises au niveau européen. La question de la concurrence internationale est souvent conjuguée à la fois au développement d'une "éducation tout au long de la vie ", à la promotion d'une société de la connaissance pour tous et à la construction d'un espace européen de l'enseignement supérieur ${ }^{2}$ (Ministère de la Recherche, 2002, volet 1).

6 Partant d'un tel contexte et de tels enjeux, quelle place les textes des appels à projet accordent-ils à la question des usages? À travers ceux-ci, peut-on identifier ce que les initiateurs de l'opération attendent des projets proposés?

\section{Capter les représentations}

7 Force est de constater que la question des usages ${ }^{3}$ occupe une place de choix dans le discours qui accompagne l'opération Campus numériques. Ceux-ci sont, en effet, présentés comme étant au centre des préoccupations des initiateurs. L'objectif " global » du développement des campus étant « d'offrir un cadre d'apprentissage innovant s'appuyant sur les technologies numériques », les usages sont le moyen d'y accéder, comme en témoigne cet extrait de la lettre adressée par le Ministère aux membres du jury chargés de sélectionner les campus : "Cela suppose le développement et la mise en œuvre mutualisés de dispositifs et de services innovants, centrés sur l'étudiant et ses besoins : formations modularisées, suivi individualisé, prise en compte du nomadisme, etc. " (Ministère de l'Éducation, 2002). Moins que la mise en place de nouvelles technologies, il s'agit surtout par ces usages de rénover "les enseignements et la vie universitaire » (idem). Et c'est à cette aune que seront sélectionnés les projets.

8 Trois constats, complémentaires, se dégagent alors. Nous sommes en présence d'un positionnement du Ministère en tant que porte-parole des usagers potentiels (le corps enseignant, les étudiants et les personnels administratifs et techniques des universités) pour exprimer les intérêts, les représentations ou les demandes latentes de ces derniers, ce que P.Chambat (1994, p. 48) désigne comme une captation des représentations (premier constat). Il existe aussi une anticipation des usages, indispensable pour que les initiateurs puissent légitimer leur opération alors qu'il leur est difficile de prédire les usages réels des dispositifs à mettre en place (deuxième constat). Enfin, à ce stade de développement, ne peuvent être formulées que des prescriptions générales d'utilisation (troisième constat).

9 Marquées par une grande instabilité dans les orientations prises sur le court terme, ces prescriptions générales d'usages ont fait l'objet de plusieurs changements dès 2002 (année du troisième appel à projet). En effet, si les deux premiers appels avaient comme objectif le développement de l'enseignement à distance avec une logique guidée par l'expérimentation, le troisième affichait une démarche sensiblement différente marquée par le passage à une logique d'action guidée par l'idée de généralisation. L'objectif est devenu la mise à disposition pour les établissements d'enseignement supérieur d'une offre diversifiée de solutions technologiques d'espaces numériques de travail. À côté des consortiums dédiés à l'enseignement à distance (volet 1), apparaît une nouvelle forme organisationnelle : l'espace numérique de travail (volet 2). Puis, peu de temps après, la politique ministérielle, motivée par la décentralisation, change de cap avec les universités numériques en région (décembre 2002) ${ }^{4}$, et enfin les universités numériques thématiques, c'est-à-dire des consortiums regroupés par discipline. De tels changements dans les 
orientations rendent peu lisibles les actions ministérielles en matière d'intégration des TIC et de prescriptions d'usages.

\section{Impliquer la recherche en sciences sociales}

10 L'importance accordée à la question des usages et des pratiques par les initiateurs de l'opération ne se limite pas à une production discursive. Cette question semble être un élément structurant de la politique de la sous-direction des Technologies en ce qu'elle constitue à elle seule un programme d'action ${ }^{5}$ visant « à mettre à la disposition de la communauté éducative l'infrastructure et les services adaptés au développement des usages " (Ministère de l'Éducation, site Tice Educnet, 2006). Il est question d'appuyer l'expérience par la mise à disposition d'études sur les technologies et leurs usages en associant les chercheurs en sciences sociales et d'identifier les usages potentiels qui se dégagent des différents projets proposés ou déjà lancés. Des séminaires sont organisés pour reprendre des problématiques telles que le nomadisme ou le suivi personnalisé des étudiants; des rapports sont produits dans ce sens; des observatoires des usages (comme la base de données de l'ORME ${ }^{6}$ ) sont cités en exemple; plusieurs programmes de recherche sont lancés ${ }^{7}$. Bref, l'objectif affiché est d'enclencher "une réflexion transversale [...] sur les pratiques des TIC [devant] permettre de constituer progressivement une banque de données des 'bonnes pratiques' de celles-ci » (Ministère de l'Éducation, 2006, site Educnet, mai 2006).

11 Cette sollicitation de la recherche en sciences sociales s'inscrit dans une tradition des pouvoirs publics qui «ne se satisfont plus du mélange de discours techniciste et de prophétie qui caractérise trop souvent les revues professionnelles " comme le souligne P. Chambat (1994, p. 45). Néanmoins, précise-t-il, cet intérêt pour la recherche en sciences sociales reste timide. Et de fait, c'est ce que nous observons. En effet, parallèlement à la mobilisation des chercheurs, le Ministère a recours à des enquêtes confiées à des cabinets d'audits ou de sondage. qui ont pour rôle de " mesurer les effets directs et indirects imputables aux actions entreprises [...], leur conformité aux objectifs et à évaluer la performance ou la valeur ajoutée des moyens mobilisés " (Ministère de l'Éducation, site Tice. education, mai 2006). Il s'agit encore d'évaluer "les effets" et "l'impact» des campus numériques sur l'offre de formation, de "mesurer la satisfaction». Cette démarche reste cependant timide et limitée, car ces notions sont en effet "éloignées de la manière dont les chercheurs abordent les usages» (idem, 1994, p. 45). Qui plus est, observer les usages ne peut se résumer à une simple mesure de satisfaction fondée sur une démarche quantitative. Il faut aussi s'interroger sur le rôle central joué par les usagers dans ce processus et analyser le plus finement possible les choix, les orientations techniques et politiques, cerner les enjeux qui en découlent. En somme, il est nécessaire d'envisager l'insertion des TIC dans les secteurs de l'éducation et de la formation dans toute leur "complexité» (Miège et alii, 2001, p. 50).

\section{Traduire des orientations politiques}

12 Il n'est pas possible d'analyser les usages ainsi préconisés sans les rapprocher d'orientations politiques à la fois supranationales et nationales. À cet égard, le texte de l'appel à projet mentionne clairement par exemple, que «la construction de l'espace européen d'enseignement supérieur est un objectif auquel l'offre de formation développée doit contribuer » (Ministère de la Recherche, 2002). Parmi les autres conséquences attendues, 
citons encore le développement de la formation continue et, plus largement, de la formation tout au long de la vie. Ces orientations, nous les trouvons aussi mentionnées au niveau européen, dans les objectifs stratégiques pour les systèmes éducatifs. Il y est question à la fois "d'améliorer la qualité et l'efficacité des systèmes d'éducation et de formation dans l'Union européenne », d'y "faciliter l'accès de tous » et de les « ouvrir au monde extérieur » grâce notamment à l'usage des TIC (cité dans Laval et Weber, 2002, p. 125).

Complémentairement, en ce qui concerne la dimension nationale, c'est une rationalisation de la formation par l'intégration des TIC qui est recherchée. Il va s'agir, par exemple, d'« optimiser les parcours et les temps de formation des étudiants » (Ministère de la Recherche, 2002a). Dans une certaine mesure, suivi individualisé, mutualisation de dispositifs et de services, formations modularisées ou encore nomadisme, renvoient à une logique de rationalisation de la formation, d'augmentation de la productivité et de réduction des coûts, dans une forme empruntée au modèle entrepreunarial (Moeglin, 2003). Les nouveaux dispositifs ${ }^{8}$, comme les nouveaux services, annoncent une pratique managériale où les futurs offreurs sont invités à répondre à un supposé besoin des étudiants et à favoriser leur émancipation.

\section{Prémices d'une réalité}

Notre intérêt va se centrer maintenant sur la façon dont la question des usages et des usagers a été abordée par les acteurs des campus : dans quelle mesure ceux-ci se sont-ils inscrits dans les représentations ministérielles lors des phases d'expérimentation et de réalisation? Les usagers visés sont-ils les usagers désignés par les porteurs de projet (étudiants de formation initiale, étudiants de formation continue et étudiants à l'étranger)? Et retrouve-t-on ces trois catégories comme usagers réels des campus? Incontestablement, ces étudiants existent puisque déjà inscrits dans une des nombreuses écoles et universités partie prenante de ces campus. Mais cela n'en fait pas pour autant «naturellement » des utilisateurs des campus. Tenter de répondre aux questions posées, c'est chercher encore une fois à contextualiser chacune des expériences, et ceci à deux niveaux :

- celui de la mise en perspective des origines fondatrices des différents projets ;

- celui des intentions initiales des porteurs de ces projets et de leur évolution: publics auxquels ils souhaitent proposer leur offre, ajustements auxquels ils ont dû procéder pour prendre en compte la demande des publics effectifs et pour faire face aux difficultés inhérentes au contexte d'expérimentation.

\section{Tenir compte des initiatives antérieures}

Lorsque le premier appel à projet est lancé au printemps 2000, de nombreux acteurs inscrits dans de multiples réseaux y voient l'occasion de faire reconnaître des initiatives en cours, déjà anciennes pour certains. Par exemple, il faut remonter à 1987 pour identifier le «moment fondateur » de C@mpusciences ${ }^{9}$ (Combes, Moeglin, 2005, p. 17). C'est à cette date, en effet, que le Réseau universitaire des centres d'autoformation (RUCA) et la Fédération interuniversitaire de l'enseignement à distance (FIED) sont reconnus par le ministère de l'Éducation nationale, de la jeunesse et des sports. Un peu plus tard, ces deux entités se rejoignent au sein du Groupement pour l'enseignement sur mesure médiatisé (GEMME). Dans ce sens, C@mpusciences est la «dernière manifestation en date» de ces 
évolutions (op.cit., p. 18). La preuve en est que, à la différence de la plupart des autres campus numériques, celui-ci «n'a pas pour objectif principal de produire des contenus numérisés " puisque ceux-ci sont déjà disponibles, ayant été produits par le programme Premier cycle sur mesure (PCSM) mis en place par le RUCA. La priorité est donc bien plus de favoriser l'accès à ces contenus. Et ceci n'est pas une mince affaire, car malgré la filiation établie entre RUCA et C@mpuSciences, le «modèle d'élaboration et d'utilisation des ressources numériques qui a prévalu» dans un premier temps est à cette nouvelle étape remis en cause (op. cit., p. 15).

La préexistence de réseaux, fédérations ou autres groupements fondateurs est une caractéristique que nous retrouvons aussi dans Canège ${ }^{10}$. A partir de 1999, avant que le campus ne soit désigné en tant que tel par le Ministère, lors du premier appel d'offre, et qu'il ne soit «conforté » par le contrat d'établissement 2001-2004 de l'Université ParisDauphine, un travail important avait déjà été effectué par le centre d'ingénierie pédagogique, qui lui-même faisait suite à la cellule «Innovation pédagogique » mise en place par Michel Armatte en 1995 (Grevet, 2005, p. 7).

17 Dans la généalogie des campus, on retrouve par ailleurs facilement des collectifs créés de manière ad hoc pour faire face à des questions qui se posaient. Par exemple, le groupe Platformad, à l'origine également de Canège, a été réuni afin de construire «un support pédagogique protéiforme» (op.cit., p. 12). Qui plus est, il n'est pas rare que, derrière ces collectifs, œuvrent finalement quelques individus seulement, mais dont l'implication est telle qu'elle a un effet d'entraînement. Il ne s'agit pas ici de réduire le développement d'un campus à une seule personne, mais de reconnaître le rôle moteur que certains ont joué notamment dans le passage d'une structure à l'autre. Toujours dans Canège, Michel Armatte a tenu ce rôle en promouvant et développant la même idée, comme un fil rouge, tout au long des différentes étapes. Il est en quelque sorte le lien entre le campus d'aujourd'hui et les collectifs d'hier.

De nombreux autres éléments significatifs, tirés de certaines monographies, peuvent encore nous éclairer sur tel ou tel aspect des usages ou des usagers. La suite de notre article traitera essentiellement de deux cas, CampusCultura et C@mpusciences ${ }^{11}$, ce choix se justifiant par la préexistence de projets qui ont été à leur source, mais aussi par l'intérêt et les divergences de représentations qu'a suscités la question des usages auprès des acteurs impliqués ainsi que son évolution tout au long des projets.

\section{Produire des contenus médiatisés : quelle place pour l'usager?}

19 Du côté des acteurs des campus, la question des usages est d'importance, mais son appréhension s'avère incontestablement plus délicate, car c'est à ce niveau que les ressources sont confrontées aux utilisateurs et que l'on découvre en pratique les usages possibles des dispositifs mis en place ainsi que leurs limites, voire leur inadéquation avec les besoins de ceux à qui ils s'adressent. Ainsi, deux cas de figures se présentent à nous : la question de la médiatisation a été pensée en même temps que la production de contenus, ou bien elle est posée après la production de ceux-ci. Le premier cas est certainement le plus séduisant car cela signifie que le module terminé est prêt à l'usage. Cependant, pour y arriver, il a fallu bien souvent en passer par toute une série d'arbitrages assurément dévoreurs de temps, mais qui portent sur les fondations mêmes du campus; par exemple, sur la définition du contenu du module, sur les images et photos utilisées, sur le séquençage, sur le codage ou encore sur la scénarisation. Bien évidemment, ces micro- 
décisions à prendre dès la production du premier module retardent d'autant sa production et donc son utilisation. Cependant, elles permettent aussi d'accumuler de l'expérience et de gagner du temps sur la production des modules suivants. Autrement dit, deux risques s'opposent entre lesquels il est nécessaire de trouver une voie médiane : le premier est de produire d'abord des modules sans se préoccuper des problèmes afférents (la question des droits, la question de la rémunération des auteurs), le second est de ne commencer à produire que lorsque ces questions sont réglées. Pour résumer, le plus court chemin vers l'utilisation des modules, et donc vers les utilisateurs, risque de conduire à des blocages importants. Or, il convient de ne pas oublier l'objectif des campus, à savoir la mise à disposition de contenus numérisés pour des usagers et, dans les deux cas, les arbitrages doivent tenir compte de cet impératif.

Par exemple, les initiateurs du CampusCultura ont choisi de mener de front à la fois le développement des ressources et celui de la plate-forme. L'objectif affiché est double: respecter les délais de mise en ligne et arriver à maitriser les coûts globaux de production des ressources et de leur médiatisation. Ainsi, le chef de projet, Odile Coppey, peut-elle avancer : "C'est 12 à $15000 \mathrm{~F}$ la médiatisation d'une heure de formation sur le marché et nous on arrive à médiatiser des heures de formation à $5000 \mathrm{~F}$ » (Fichez, Benchenna, 2005, p. 34). Autour de ce campus, un groupe de travail, composé d'experts de la médiation culturelle, d'enseignants et un prestataire de service dans le développement multimédia (CybEOsphere) s'est constitué. Son rôle a été de déterminer l'architecture pédagogique des modules, d'en décrire le contenu et de proposer une scénarisation, confiée en majorité à ce prestataire. En s'arrêtant sur l'analyse du processus de médiatisation des cours, on s'aperçoit que l'accent est mis sur la production et que l'usager passe au second plan. Il n'est ainsi impliqué que lorsque la ressource est médiatisée et mise en ligne ${ }^{12}$.

21 Mais d'un campus à l'autre, les situations sont bien différentes et il n'a pas toujours été possible d'aborder la question de la médiatisation de la sorte, comme le montre le second cas de figure. S'appuyant sur un acquis déjà très important en matière de production à la date du lancement de l'appel à projet du Ministère, certains ont voulu relever la gageure de scénariser ces contenus afin de les inscrire davantage dans la logique d'usages des campus numériques favorisant un accès prioritairement à distance des cours. Mais les difficultés ont alors été doubles, comme l'ont relevé Y. Combes et P. Moeglin : il s'agit alors d'« inscrire dans la logique de l'apprentissage des ressources conçues selon la logique des matières à enseigner, en mettant ces ressources au service d'usages déployés dans d'autres contextes que ceux où elles ont été produites et sont utilisées au départ »; il s'agit aussi de " concevoir un mode flexible et ouvert d'accès aux ressources, en lieu et place du mode concentré et hiérarchisé qui a guidé leur production », de "substituer à l'organisation de la filière par l'amont une structuration soumettant la conception et la prestation de l'offre aux orientations de la demande » (2005, p. 14).

Autrement dit, les difficultés sont d'une part pédagogiques et techniques, et d'autre part organisationnelles. Accessoirement, à travers elles, se joue une transformation de la figure de l'usager (de récepteur d'un cours générique à utilisateur de services pédagogiques sur-mesure dans lequel le cours proprement dit n'est plus qu'un élément parmi d'autres) et d'un déplacement du processus d'industrialisation (de l'étape de production à l'étape de médiation). 


\section{Concevoir l'usager : des hésitations aux certitudes}

23 C'est dans le déroulement des projets, les intentions et les convictions initiales de leurs porteurs, les temps forts qui les ponctuent, les contradictions entre les points de vue des acteurs impliqués que l'on peut identifier la façon dont a évolué la prise en compte des usages et des usagers. Ainsi pour C@mpusciences, Y. Combes et P. Moeglin montrent comment la conception de l'usager est passée par des hésitations entre plusieurs approches. Si l'usager était au cœur du projet RUCA, il est mis au second plan lors de la phase de production des ressources (entre 1996 et 2000) pour redevenir central dans le projet C@mpusciences. Les auteurs identifient ainsi trois temps forts où deux conceptions de l'usager, et donc des usages, vont chercher à s'imposer. Dans un premier temps, les initiateurs du RUCA (émanant de la formation continue) mettent l'accent sur la personnalisation de la formation, la responsabilisation de l'apprenant et son épanouissement personnel. Dans un deuxième temps, consacré plus particulièrement au développement des ressources pédagogiques, l'approche de l'usager s'oriente vers une disqualification de la priorité pédagogique initiale. Ainsi, l'usager est appelé à s'adapter à la progression prescrite par le programme et à s'inscrire dans une approche traditionnelle de la logique des matières à enseigner. Mais l'autoproduction des ressources et l'autoconsommation relative à celles-ci n'entraînent pas d'usages élargis à des publics différents (formation initiale, formation continue, enseignement à distance) ${ }^{13}$. Cet état de fait va conduire les acteurs à repenser la question des usages dans le cadre du campus, en saisissant l'opportunité du premier appel à projet (2000). L'idée de pilotage de l'offre pédagogique par la demande refait alors surface, et la place de l'usager fait l'objet de confrontations entre deux modèles extrêmes : l'usager se conformant au programme proposé en formation initiale avec une pédagogie de type transmissif (les ressources sont construites de manière linéaire; l'usager s'inscrivant dans une pédagogie appropriative où les ressources sont mobilisées à son service et où l'ingénierie de médiation est privilégiée à l'ingénierie de production.

Pour les deux auteurs, la création du campus et les fluctuations de ses orientations seraient liées au passage d'une innovation managériale (contexte Université en ligne/ RUCA) à une innovation potentiellement instauratrice d'un nouveau paradigme éducatif (autre manière d'enseigner). Ces transformations se traduisent par un double processus : une disqualification du modèle de production de ressources pédagogiques ex-ante et l'élaboration d'un modèle privilégiant la mise à disposition de ressources modularisées et indexées à la demande.

Si la question des usages a connu des fluctuations dans le cas précédent, la place de l'usager "rêvé »" pour les initiateurs du CampusCultur@ faisait non seulement l'objet d'un accord pour tous les partenaires mais constituait aussi un objectif poursuivi et mis en œuvre de longue date. L'appel à projet campus numériques était, entre autres, une opportunité à saisir afin de développer de nouvelles modalités pour former aux métiers de la médiation culturelle en s'appuyant sur les TIC. Le public prioritaire du campus était celui de la formation continue du secteur de la médiation culturelle et plus particulièrement les emplois jeunes, en demande de qualification professionnelle, favorisée par des dotations ministérielles. Mais, au bout de la première année d'existence, des divergences de représentations et d'enjeux autour des compétences à mettre en œuvre dans un dispositif de formation professionnelle déclenchent une crise entre les 
protagonistes qui se solde par le retrait du porteur du projet, l'Université d'Avignon. Précisément, ce conflit se matérialise en partie par des interrogations sur le statut des universitaires et des professionnels de la médiation culturelle participant au projet et sur la place professionnelle qu'ils occupent. Deux visions sociétales du rôle de l'université et de son rapport au monde professionnel sont ainsi au cœur des divergences. Le point d'achoppement porte sur le positionnement et le rôle que doit occuper l'institution dans l'élaboration, la pérennisation et la formation des usages d'un campus numérique destiné aux futurs professionnels des métiers de la médiation culturelle.

Pour autant, cette tension n'affectera pas l'ouverture effective du campus en direction d'un double public (formation continue et formation initiale) ${ }^{15}$. Il semble même que les résultats des étudiants aient été meilleurs après qu'ils aient eu accès en ligne à ces ressources ${ }^{16}$, et qu'ils aient perçu en tout cas le campus comme un «dispositif de formation efficace et pratique " (Fichez, Benchenna, 2005). Cette satisfaction porte, notamment, sur les éléments interactifs (animations, quizz) qui «sont appréciés parce qu'ils offrent des voies d'accès différentes aux savoirs exposés et qu'ils concrétisent les intentions pédagogiques des enseignants », sur le forum général du campus qui «est visité de façon régulière par une majorité d'étudiants qui contribuent peu mais se disent très attachés à sa fonction de mutualisation des pratiques ", sans oublier les cours eux-mêmes qui sont globalement «bien faits, riches et variés » (voir l'évaluation réalisée par Pouts-Lajus et Leccia, 2004, p. 33).

Le problème n'en reste pas moins celui de la pérennisation du campus du fait des crises qu'il a connues et du rétrécissement répété du nombre de partenaires. En l'état, cela rend difficile toute conjecture sur la stabilisation des usagers et des usages.

\section{Conclusion}

Comme nous l'avions annoncé, traiter des usages dans le cadre de l'opération Campus numériques français s'avère, pour le moment, une gageure. À l'heure actuelle, les campus labellisés continuant à fonctionner entrent à peine dans une phase opérationnelle où il est encore difficile d'identifier des modes récurrents d'utilisation et relativement intégrés dans la quotidienneté. Pourtant, il a été possible de chercher à comprendre les intentions de tout un ensemble d'acteurs (initiateurs du programme, porteurs des projets, usagers) par une analyse des discours accompagnateurs de l'opération et des actions les accompagnant, ainsi que par celle des premières traductions concernant les intentions des acteurs. Au terme de notre réflexion, nous retenons trois points :

- le discours ministériel accompagnant l'intégration des TIC dans le système éducatif français en général, et l'opération Campus numériques en particulier, accorde une place importante à la question des usages. Remplissant sa fonction de légitimation, ce discours suppose l'existence de besoins et préconise des usages en mettant en avant une série d'arguments de sphères différentes (technique, sociale, politique, éducative) ;

- cependant, on peut noter une certaine instabilité, sur le court terme, des orientations ministérielles. En effet, les prescriptions générales d'usages, identifiées au début de l'opération, ont fait l'objet de changement d'orientation dès 2002 ;

- enfin, un regard porté sur deux campus en particulier (c@mpuSciences et Campus Cultura), nous a éclairés sur les hésitations ou les certitudes des porteurs de projet quant à leur représentation des usagers auxquels ils souhaitent s'adresser, et quant à la place qu'ils leur accordent dans le processus de production des ressources ou la mise en place des dispositifs. 
Au final, il semble que l'opération soit bien novatrice malgré tout, en ce sens qu'elle tente de mettre les usages d'une offre et les usagers au cœur des préoccupations. Incontestablement, l'entreprise est difficile, plus sans doute que de produire des ressources! Si bien qu'on peut avancer l'idée que le projet de mise en place de campus numériques est, pour le moment, plus intéressant pour ce qu'il a engendré comme mouvement (coopérations entre acteurs, arbitrages réalisés sur certains points) que pour ce qu'il a produit comme ressources et dispositifs. En l'état, il faut alors avouer que c'est moins à l'usage des campus eux-mêmes que nous nous sommes intéressés, qu'à l'usage du projet dans le contexte français.

\section{BIBLIOGRAPHIE}

CampusCultura, (2001), Campus numérique, appel à projets 2001, volet 1, formulaire de candidature: Cultura, Université d'Avignon et des Pays du Vaucluse, Avignon, juin 2001.

Chambat, P., (1994), « NTIC et représentations des usages », in : Vitalis A., Médias et nouvelles technologies : pour une socio-politique des usages, Éditions Apogée, Rennes.

Combès, Y. et Moeglin, P., (2005), « C@mpuSciences : d'un modèle industriel à l'autre », accessible aux adresses indiquées à ERTe CN 2005.

ERTe CN, (2005), Résultats 2005 de l'ERTe (Équipe de Recherche Technologique éducation) « Modèles économiques et enjeux organisationnels des campus numériques ». Accessible à www.ifresi.univlille1.fr/SITE/2_Recherche/22_Programmes/ERTe/ERTe.htm ou à partir de la page d'accueil www.mshparisnord.org/.

Fichez, E. et Benchenna, A., (2005), « Le cas CampusCultura », accessible aux adresses indiquées à ERTe CN 2005.

Grevet, P., (2005), «L'expérience économico-institutionnelle de Canège », accessible aux adresses indiquées à ERTe CN 2005.

Lacroix, J.-G., Mœglin, P. et Tremblay, G., (1992), « Usages de la notion d'usage : NTIC et discours promotionnels au Québec et en France ", Les nouveaux espaces de l'information et de la communication, in : Actes du $8^{e}$ Congrès national des sciences de l'information et de la communication, Lille, mai 1992.

Laval, C. et Weber, L., (coordination de), (2002), « Le nouvel ordre éducatif mondial », Institut de recherche de la FSU, éditions Syllepse, Paris.

Pouts-Lajus, S. et Leccia, E., (2004), «Évaluation formative accompagnant la mise en place de CampusCultura (CNED), Usages et usagers », Rapport final, septembre 2004.

Mège, B., Séguy, F. et Quinton, P., (2003), « En quoi les Tice innovent-elles ? Pour une analyse communicationnelle du recours aux TIC dans l'enseignement supérieur », Actes du Colloque Bogues 2001, TIC et éducation, t. 3, Les Presses de l'Université Laval.

Ministère de l'Éducation : site www.educnet.education.fr/, Ministère de l'Éducation nationale, Paris, mai 2006. 
Ministère de l'Éducation :site tice.education.fr/educnet/public/superieur/campus, Ministère de l'Éducation nationale, Paris, mai 2006.

Ministère de l'Éducation : Lettre aux membres du jury de l'appel à projet 2002, Sous-direction des Technologies, Ministère de l'Éducation nationale, Paris, 18 juin 2002.

Ministère de la Recherche : Campus numérique, appel à projets 2002 : objectifs et modalités, Ministère de la Recherche et Ministère de l'Éducation nationale, Paris, mars 2002.

Ministère de la Recherche : Campus numérique, appel à projets 2002 : volet 1 , Ministère de la Recherche et Ministère de l'Éducation nationale, Paris, mars 2002.

Ministère de la Recherche : Campus numérique, appel à projets 2002 : volet 2, Ministère de la Recherche et Ministère de l'Éducation nationale, Paris, mars 2002.

Moeglin, P., (2003), « Industrialisation de la prestation éducative, de la médiatisation à la rationalisation ", Actes du Colloque Bogues 2001, TIC et éducation, t. 3, Les Presses de l'Université Laval.

Thibault, F., (2003), « Coalitions sociales et innovation pédagogique : le cas du Réseau Universitaire des Centres d'Autoformation », in : Autoformation et enseignement supérieur, B. Albéro (dir.), Paris, Hermès Science/Lavoisier.

\section{NOTES}

1. Voir l'adresse électronique http://www.educnet.education.fr/superieur/archives/ campus2000.htm.

2. Ainsi, dans l'appel à projet 2002 du Ministère, on peut lire : "Dans un contexte de concurrence internationale accrue, les ministères de l'Éducation nationale et de la Recherche, soucieux de préserver l'excellence de l'enseignement supérieur et de le moderniser, ont lancé successivement, en 2000, 2001 et 2002, trois appels à projets pour la constitution de 'Campus numériques français'. L'objectif majeur des appels à projets était d'arriver à construire une offre nationale de formation ouverte et à distance (FOAD) de qualité et compétitive sur le marché international. Avec le développement de l'éducation tout au long de la vie, les besoins de l'Enseignement Supérieur se sont extrêmement diversifiés " (Ministère de la recherche, 2002, volet 1, p. 1).

3. Si la littérature sur les usages fait la distinction entre les termes d'usage et de pratique, les deux sont utilisées ici indifféremment : voir notamment J. Jouët dans Dictionnaire critique de la communication, L. Sfez (sous la direction de), P.U.F., Paris, 1993, p. 371.

4. http://www.educnet.education.fr/superieur/unr.htm, page consultée le 15 octobre 2006.

5. À côté de quatre autres programmes d'action qui sont : Infrastructures et services, Ressources numériques, Formation et Qualité : voir le site http://www.educnet.education.fr/sections/plan/ reseau8982/ (consultation, mai 2006).

6. L'ORME se définit comme mission «d'observer l'usage du multimédia, et plus généralement des technologies de l'information dans le système éducatif»: voir le site http://www.ormemultimedia.org/ (consultation, mai 2006).

7. http://www.tematice.net/index.php?rub=4\&srub=25 : nous pouvons citer l'ACI Formation et Education ; Erté ; programme Incitatif de Recherche sur l'Éducation et la Formation (PIREF) ; Plan d'action TICE 2003-2006 du Ministère de l'Éducation Nationale français.

8. Yolande Combès a montré que les formes d'indexation et de modularisation ou de granularisation «tentent d'ajuster les dispositifs en fonction des besoins » (« Penser les mutations des télé-services info-communicationnels: approches et perspectives », Rapport d'habilitation, Université Paris 13, Paris, décembre 2004, p. 36). 
9. Une formation ouverte à distance en mathématiques, physique, chimie et informatique.

10. Une formation ouverte à distance en économie et en gestion.

11. Nous ne nous réfèrerons qu'à une partie seulement de ces monographies réalisées dans le cadre de l'ERTé, car, faut-il le préciser, l'objet de la recherche était bien plus l'analyse des modèles économiques et des enjeux organisationnels des campus que l'analyse des usages et des usagers.

12. C'est ainsi qu'une étude commanditée par le CNED souligne que les étudiants sont gênés par l'hétérogénéité des cours en ligne lorsqu'elle affecte certains éléments de présentation ou de navigation : voir (Fichez, Benchenna, 2005).

13. Ce manque d'utilisation est justifiée à la fois par le fait que les enseignants non producteurs des ressources éprouvent des difficultés à se les approprier et par le fait que les ressources ellesmêmes, structurées en un ensemble, se prêtent peu au découpage en modules. Ainsi, le produit est-il perçu comme trop académique par certains étudiants (formation continue).

14. Table ronde "Campus numérique : première tentative de bilan scientifique " dans le cadre des $11^{\mathrm{e}}$ rencontres de l'Orme, Marseille, 2006, http://rencontres.orme-multimedia.org/Campusnumerique-premiere, $154 . \mathrm{html}$.

15. Les premiers ont accès aux ressources et aux services les accompagnant (tutorat, correction des devoirs, etc.) à la différence des seconds qui ont seulement accès aux ressources.

16. Voir entretien avec Jean-Charles Bérardi (Fichez, Benchenna, 2005).

\section{RÉSUMÉS}

À défaut de constater de véritables usages des campus numériques, les auteurs cherchent, dans un premier temps, à identifier comment cette question est abordée par les initiateurs. Tout en notant une certaine instabilité des orientations ministérielles sur le court terme, ils tentent de montrer comment les usages projetés peuvent être compris comme une traduction d'exigences politiques à la fois nationale et supranationale. Remplissant sa fonction de légitimation, le discours ministériel suppose l'existence de besoins et préconise des usages, mettant en avant une série d'arguments de sphères différentes (technique, économique, politique, éducative). Dans un second temps, ils focalisent leur analyse sur l'importance accordée aux usages et usagers, tant au niveau des réponses aux appels dans les consortiums constitués qu'à celui des prémices d'une réalité en émergence, en portant leur regard plus particulièrement sur la représentation qu'ont des usagers les porteurs de deux campus, C@mpuSciences et CampusCultura.

In the first part of the article, the authors attempt to identify, in the absence of any evidence of real use of digital campuses, how this question is addressed by its initiators. Even though they note a certain degree of instability in the short term orientations chosen by the government, the authors seek to demonstrate how the projected uses can be seen as translating political demands from both a national and supranational level. The ministerial discourse fulfils its legitimising role by identifying supposed needs and uses, emphasising a series of arguments coming from various spheres (technical, economic, political and educational). In the second part, the authors focus their analysis on the importance accorded to uses and users, with respect to both the response to invitations to participate in the constituted consortiums and to an emerging reality, by studying in particular how users are represented by those responsibles for two campuses, C@mpuSciences and CampusCultura. 
INDEX

Keywords : uses, users, innovation strategies, public action, digital campus

Mots-clés : usages, usagers, innovation, politique publique, campus numérique

\section{AUTEURS}

\section{ABDEL BENCHENNA}

Abdel Benchenna est maître de conférences en sciences de l'information et de la communication à l'université Paris 13. Membre du LabSIC et du Séminaire Industrialisation de la Formation, ses recherches portent sur les mutations, en cours, des secteurs de l'éducation et de la formation, tant au Maghreb qu'en France. Adresse électronique : abdel.benchenna@wanadoo.fr.

\section{VINCENT BRULOIS}

Vincent Brulois est maître de conférences en sciences de l'information et de la communication à l'Université Paris 13, attaché au LabSIC et à la MSH Paris Nord. Concernant les campus numériques, il participe à une recherche, pilotée par le GRESEC de l'Université Stendhal Grenoble 3, portant sur une approche communicationnelle de produits technologiques destinés à l'enseignement et sur leurs conditions de production. Adresse électronique : brulois@sic.univparis13.fr. 